\title{
Efficacy and Safety of Proton Therapy for Medulloblastoma Patients: A Mata-Analysis
}

\author{
Wenyue Deng ${ }^{1}$, Mingyang Liu ${ }^{1}$, Shiyuan Jing ${ }^{2}$, lingling Zhang ${ }^{3}$, Xuhui Liu ${ }^{3}$, Weiping Zhan ${ }^{3 *}$ \\ ${ }^{1}$ Department of Neurosurgery, Wuhan Children's Hospital(Wuhan Maternal and Child Healthcare Hospital), Tongji Medical College, Huazhong \\ University of Science \& Technology, Wuhan, China.
}

${ }^{2}$ Department of Neurosurgery, Sanbo Brain Hospital, Capital Medical University, Beijing, China.

${ }^{3}$ Department of pediatrics, Laizhou Maternal and child Health care hospital, Yantai, China.

*Corresponding Author: Weiping Zhan, Spinal Unit, Department of pediatrics, Laizhou Maternal and child Health care hospital, Yantai, China.

Received date: October 15, 2021; Accepted date: November 04, 2021; Published date: January 04, 2022

Citation: Wenyue Deng, Mingyang Liu, Shiyuan Jing, lingling Zhang, Xuhui Liu, Weiping Zhan. (2022) Efficacy and Safety of Proton Therapy for Medulloblastoma Patients: A Mata-Analysis. J. Neuroscience and Neurological Surgery. 11(2); DOI:10.31579/2578-8868/207

Copyrights: (C) 2022 Weiping Zhan, This is an open-access article distributed under the terms of The Creative Commons Attribution License, which permits unrestricted use, distribution, and reproduction in any medium, provided the original author and source are credited

\begin{abstract}
Background: Currently, photon therapy is mainly used for radiotherapy, however, the long-term side effects of photon therapy are sometimes reported, especially in children, including cognitive decline, mental decline, growth retardation, endocrine dysfunction and secondary tumor.Compared with photon radiotherapy, proton therapy is a novel method of radiation therapy, which reduces acute and late radiation damage and improves patients' quality of life. Therefore, we evaluated proton therapy on medulloblastoma patients by meta-analysis in this study.

Materials and Methods: A clinical randomized controlled trial was conducted by retrieving the main databases PubMed (Medline), EMbase, Cochrane Library, Chinese Biomedical Literature Database (CBM), Chinese Zhi-wang (CNKI), Wanfang Database, and VIP Database. We analyzed the literature which matched the quality criteria for the prognostic impact of proton and photon therapy on medulloblastoma patients.

Results: Ten articles were included in this study. The overall survival (OS) rate and side effects were comprehensively analyzed. The results showed that proton radiotherapy significantly reduced the side effects and recurrence rate of tumor.

Conclusion: Proton therapy could significantly reduce the side effects and recurrence rate of medulloblastoma in patients.

Key words: proton radiotherapy; medulloblastoma; efficacy; safety; meta-analysis
\end{abstract}

\section{Introduction}

The current treatment strategy of medulloblastoma is surgery combined with radiotherapy and adjuvant chemotherapy [1]. The 5-year overall survival (OS) rate is close to $85 \%$ at standard risk, however, the high-risk patients are approximately 70\%[2].Compared with photon radiotherapy, proton therapy is a novel method of radiation therapy, which could accurately kill tumor cells and protect the organs around the tumor. Moreover, the therapy reduces side effects and improves quality of life. With the development of proton therapy, an increasing number of medulloblastoma patients are receiving the therapy method [2].

The study of medulloblastoma proton therapy was in conformity to the principle of randomization [3,4], mainly involving observational studies with a lower level of evidence in randomized controlled studies. This study systematically collected literature of proton therapy on medulloblastoma patients, and evaluated its efficacy and safety.

\section{Materials and methods}

\section{Methods}

\subsection{Criteria for inclusion and exclusion of literature}

1.1.1 Inclusion criteria: (1) Research types: Observational studies published in China and abroad, Chinese and English literature.(2)Medulloblastoma confirmed by pathology, without limitation in T/M staging. No contraindication to radiotherapy, unlimited age or gender. (3) The traditional photon radiation therapy as the control group, and the proton therapy as the experimental group.

1.1.2 Exclusion criteria: (1) The study type was not clearly declared, and research data was not provided. (2) Repeatedly published literature. (3) The study could not meet the inclusion criteria. (4) The sample size was less than 10 cases. (5)There were no defined outcome and efficacy 
evaluation criteria. (6) Summary, case report, lecture and review were excluded.

\subsection{Outcome indicators}

1.2.1 Primary outcome indicators: (1) OS was defined as the time interval from the date of diagnosis to date of death or the last follow-up for survivor. (2)Recurrence free survival (RFS) was defined as the time from complete remission until recurrence or the last follow-up.

1.2.2 Secondary outcome indicators: (1)Late complications: ototoxicity, neuroendocrine toxicity including neuroendocrine (growth hormone and thyroid hormone) deficits and neurocognitive (intelligence, reasoning, memory) deficits. (2)Early and late toxicity included alopecia, fatigue, anorexia, stroke, cataract, obesity, etc.

\subsection{Retrieval Strategy}

All observational studies on postoperative proton therapy of medulloblastoma patients from January 1992 to March 2020 were retrieved by computer in PubMed, EMbase, Cochrane library, CBM, CNKI, Wanfang database, and VIP database, by MeSH of "Proton Radiotherapy, Medulloblastoma, cohort studies". In addition, references, conferences and network information were manually retrieved from the related literature.

\subsection{Literature screening}

Mingyang Liu and wenyue Deng independently screened these literature, extracted and cross-checked the data. weiping Zhan included or excluded the literature, if there were inconsistencies. The substandard literature was excluded by the subject and abstract, and then further determined by reading the full text.

\subsection{Data extraction}

(1) The basic information included in the literature: study type, author, publication year, etc. (2) The basic characteristics of the subjects: sample size, age, sex, grade, resection degree, follow-up time, etc. (3)Total and fractionated doses of proton therapy. (4) Efficacy and safety outcome.

\subsection{Literature quality evaluation}

A total of 10 articles were included by rigorous screening, including 4 retrospective case-control studies and 6 observational studies. The statistical method was scientific and reasonable, and articles with complete data underwent data extraction. The Newcastle-Ottawa Scale (NOS) was used to evaluate the literatures. All retrospective cohort studies had NOS scores $\geqq 6$. The quality evaluation of high-quality literature was completed independently by shiyuan Jing. The final scores were determined by shiyuan Jing.

\begin{tabular}{|c|c|c|c|c|}
\hline Study & Selection & Comparability & Outcome & total score \\
\hline Benjamin J Moeller & 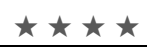 & $\star$ & $\star$ & 6 Stars \\
\hline Jimenez, R & $\star \star \star$ & $\star \star$ & $\star$ & 6 Stars \\
\hline Bree R. Eaton & $\star \star \star \star \star$ & $\star$ & $\star \star$ & 7 Stars \\
\hline Yock, T. I. & $\star \star \star \star \star ~$ & $\star$ & $\star \star$ & 7 Stars \\
\hline Bielamowicz, K. & $\star \star \star \star \star$ & $\star$ & $\star$ & 6 Stars \\
\hline Kamran, S. C. & 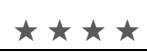 & $\star$ & $\star$ & 6 Stars \\
\hline Eaton, B. R. & $\star \star \star \star$ & $\star$ & $\star$ & 6 Stars \\
\hline Paulino, A. C. & $\star \star \star \star$ & $\star \star$ & $\star$ & 7 Stars \\
\hline Grewal, A. S. & 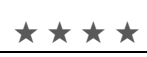 & $\star \star$ & $\star \star$ & 8 Stars \\
\hline Roshan V. Sethi & $\star \star \star \star$ & $\star \star$ & $\star$ & 7 Stars \\
\hline
\end{tabular}

Table 1: The NOS scale scores for 10 articles

\subsection{Statistical analyses}

Review Manager 5.3 and STATA12.0 software were used to analyze the data, and descriptive analyses were performed to analyze the outcome indicators that could not be combined. Firstly, the heterogeneity was evaluated. According to the Cochrane System Evaluation Guide, the heterogeneity significance level was set at $\mathrm{P}=0.1$ and $\mathrm{I}^{2}=50 \%$. When $\mathrm{P}>0.1$ and $\mathrm{I}^{2} \leqq 50 \%$, the heterogeneity between the results was not statistically significant, and the fixed effect was used to analyze the heterogeneity. When $\mathrm{P}<0.1$ and $\mathrm{I}^{2}>50 \%$, there was heterogeneity in the results. The causes of heterogeneity could be found by subgroup analysis, sensitivity analysis and meta-regression analysis. If the cause was not ascertained and the degree of heterogeneity was acceptable, random effects were selected for data consolidation. The funnel map was used to identify bias in the primary outcome.

\section{Results}

2.1 According to the retrieval strategy, 56 articles were retrieved. Based on the exclusion criteria, 10 articles were analyzed scrupulously in the study, and the document screening process was shown as follows (Figure 1). 


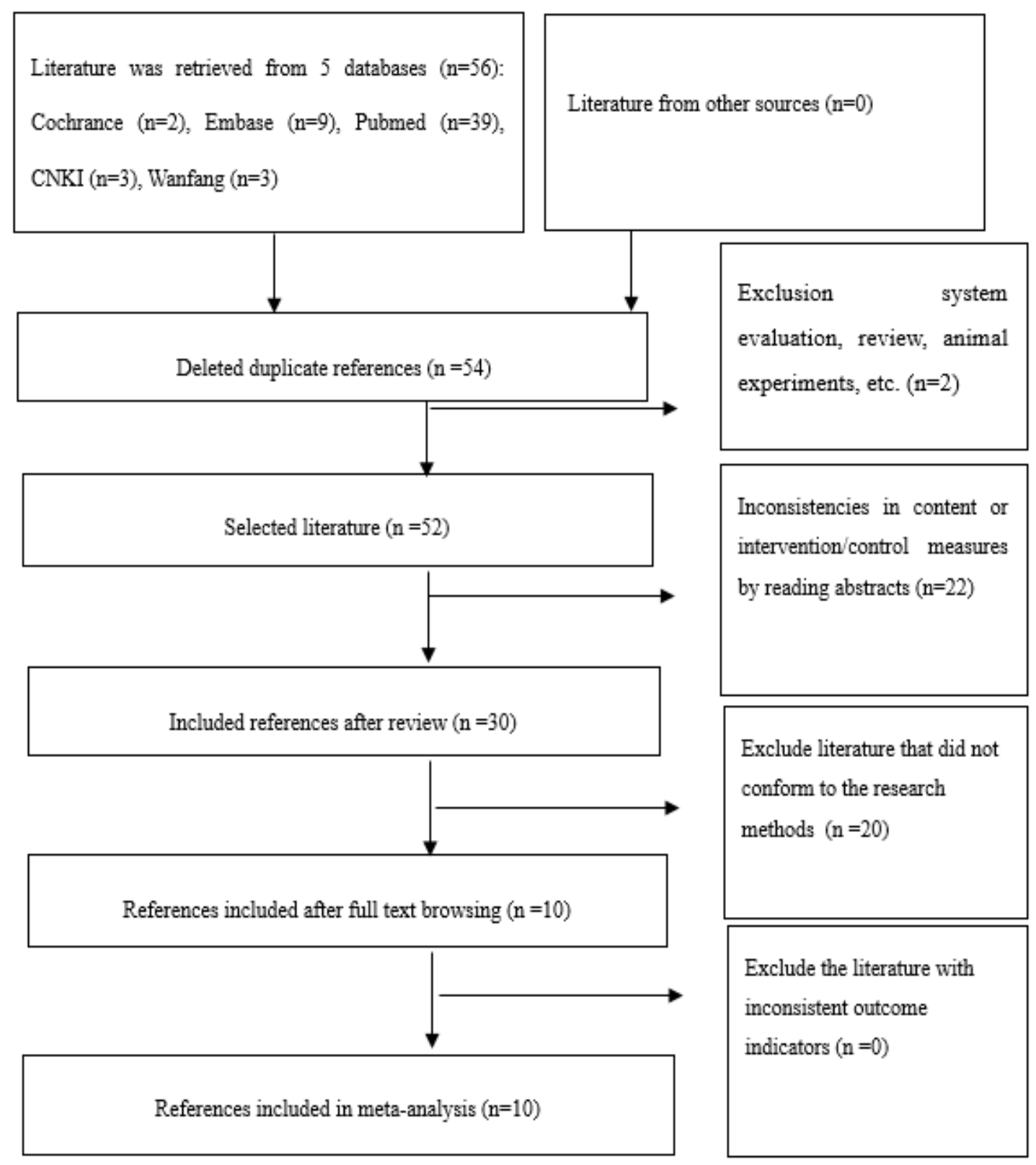

Figure 1: Document Retrieval Flowchart

\subsection{Basic characteristics of the literature}

Four articles were retrospective case-control studies, and six articles were observational studies. 492 patients were treated with proton therapy, while 180 patients were treated with photon therapy.

\subsection{Results of meta-analysis}

There was 1, 4 and 1 articles that reported 3-, 5- and 7-year OS respectively. One non-randomized controlled trial reported that 5-year OS was $82.0 \%$ for proton therapy and $87.6 \%$ for photon therapy. One observational study reported that 3-year OS was $85.6 \%$ for proton therapy, and another observational study reported that 7-year OS was $81 \%$ for proton therapy.

Firstly, the heterogeneity were examined for 5-year OS in four articles $\left(\mathrm{I}^{2}=98 \%\right.$ and $\mathrm{P}<0.1$ for $\mathrm{Q}$ test) (Figure 2 ), suggesting heterogeneity between the literature, there fore, random effects could be selected for meta-analysis. The random effects were conducted for four articles as a whole, and the results were as follows: 


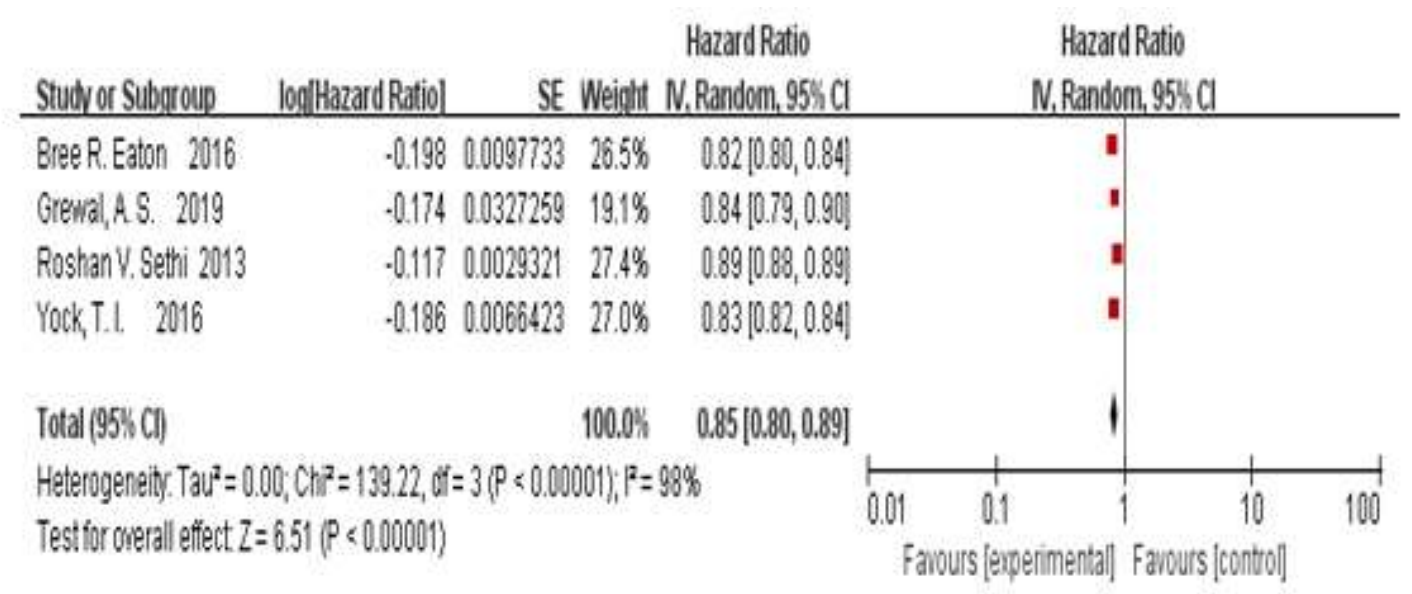

Figure 2: Forest map of 5-year survival in proton therapy

The meta-analysis showed that $85 \%$ of the total effects were statistically significant by random effects $(\mathrm{Z}=6.51, \mathrm{P}<0.05)$. The sensitivity analysis showed that anyone eliminated in the four articles would not affect the results in this study, showing that the random effects results were stable and reliable.
Meta-analysis based on random effect indicated that there was no deviation in the study, and further symmetry test showed $\mathrm{P}>0.05$, suggesting that there was no publication bias in this study (Figure 3).

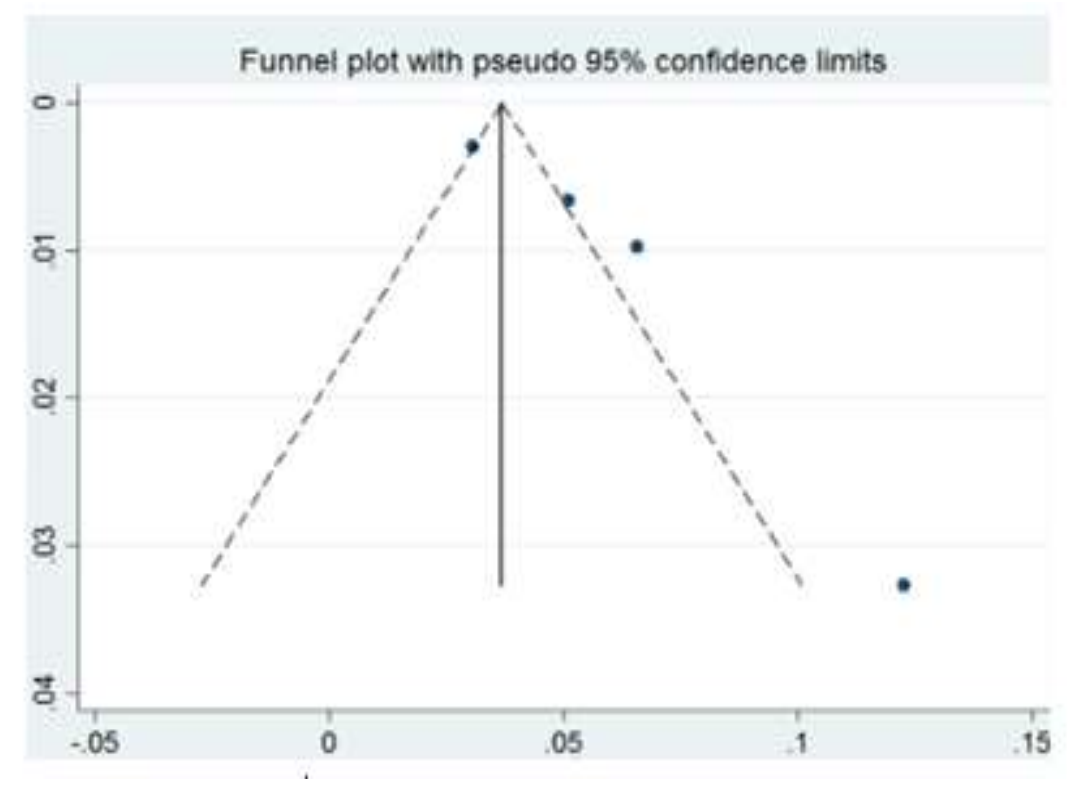

Figure 3: Funnel diagram of 5-year survival with proton therapy patients

Progression-free survival (RFS) was reported in 1 and 3 articles for 3-and 5-year OS respectively. A nonrandomized controlled trial reported that the RFS of proton therapy and photon therapy was $78.8 \%$ and $76.5 \%$ respectively for medulloblastoma. One observational study reported that the 3-year RFS of proton therapy was $83 \%$.
Firstly, 5-year RFS examined heterogeneity in three articles $\left(\mathrm{I}^{2}=0\right.$ and $P>0.1$ ) (Figure 4), suggesting no heterogeneity between the literature, and that the fixed effect could be selected for meta-analysis. Sensitivity analysis was then performed to ensure the accuracy and stability. The sensitivity analysis was carried out in three articles, and none had obvious effect on the results, revealing good stability of the study. 


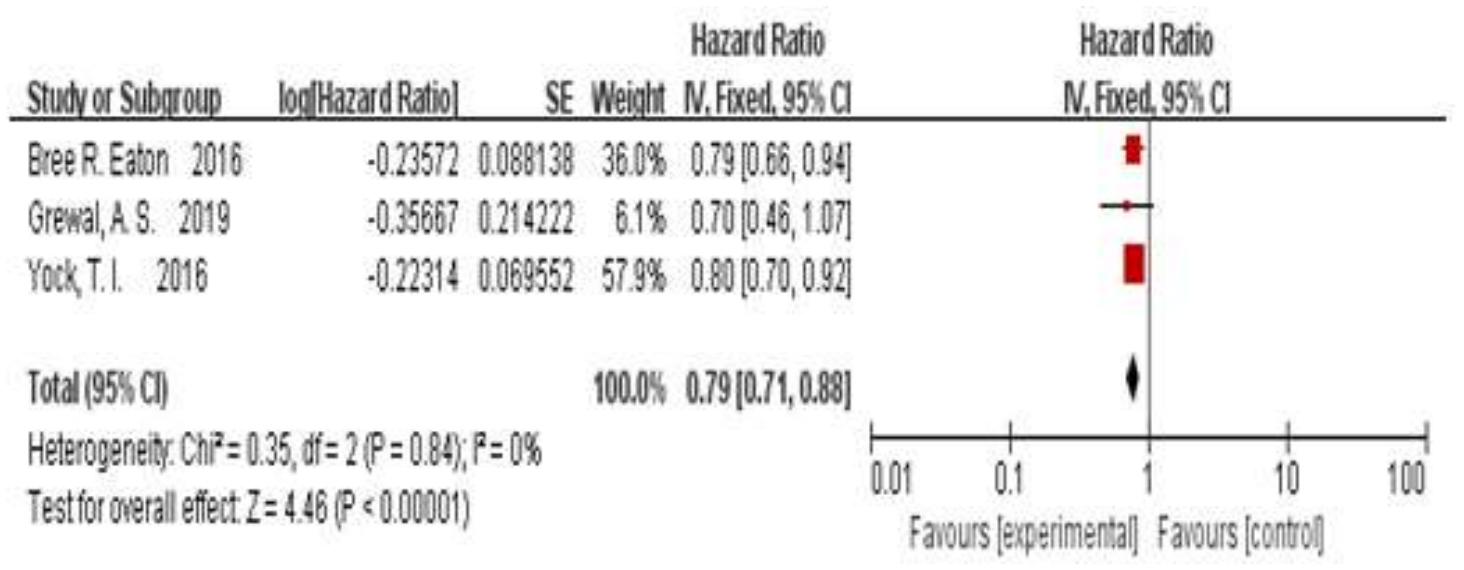

Figure 4: Forest map of 5-year RFS in patients receiving proton therapy

Meta-analysis showed that the $79 \%$ effective dose was statistically significant $(\mathrm{Z}=4.46 \mathrm{P}<0.05)$ by random effects. As shown in the following Figure, the funnel diagram was obviously symmetrical, indicating no deviation in the literature. The results of further symmetry test was $\mathrm{P}>0.05$, therefore, there was no publication bias in the following funnel diagram (Figure 5).

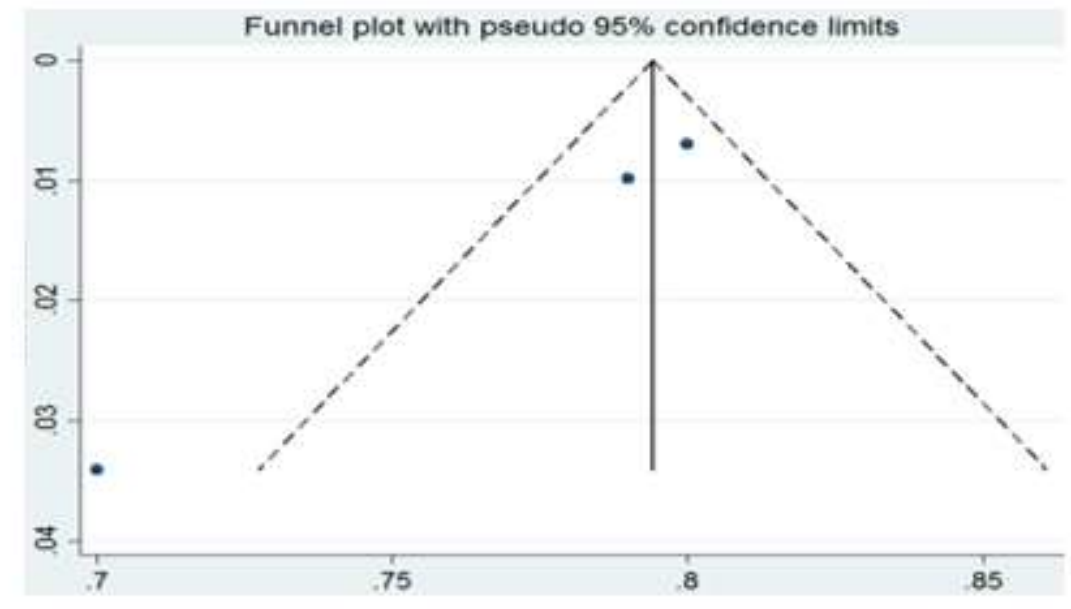

Figure 5: Funnel diagram of RFS with proton therapy

\subsubsection{Side effects}

Side effects were reported in 8 articles (Table 2), including hearing impairment $(n=27)$ and endocrine dysfunction requiring hormone replacement therapy $(n=34)$, and visual impairment $(n=5)$. Other side effects included alopecia $(n=1)$ and cataract $(n=1)$ in literature 4. Literature 5 reported a decrease in thought processing speed and speech comprehension index after proton therapy, with 1.5 points per year. There was no statistical significance in cognitive reasoning index and memory. The cumulative incidence of grade 3-4 hearing impairment was $12 \%$ at 3 years after treatment, and the cumulative incidence was $16 \%$ at 7 years after treatment Literature 6 reported that proton therapy was superior to photon therapy in central/primary hypothyroidism. Literature 7 reported 2 cases with delayed grade 2 bilateral hearing loss and 1 case with delayed grade 2 hypothyroidism.

\begin{tabular}{|l|l|l|l|l|}
\hline \multicolumn{1}{|c|}{ Study } & Sample size & $\begin{array}{l}\text { Hearing } \\
\text { impairment }\end{array}$ & $\begin{array}{l}\text { Endocrine } \\
\text { dysfunction }\end{array}$ & Visual impairment \\
\hline Benjamin J Moeller & 23 & 1 & 0 & 0 \\
\hline Jimenez, R & 12 & 8 & 3 & 1 \\
\hline Yock, T. I. & 59 & 7 & 0 & 4 \\
\hline Bielamowicz, K. & 41 & 0 & 8 & 0 \\
\hline Kamran, S. C. & 116 & 0 & 0 & 0 \\
\hline Eaton, B. R. & 40 & 0 & 22 & 0 \\
\hline Paulino, A. C. & 38 & 9 & 0 & 0 \\
\hline
\end{tabular}




\begin{tabular}{|l|l|l|l|l|}
\hline Grewal, A. S. & 14 & 2 & 1 & 0 \\
\hline Total & 343 & 27 & 34 & 5 \\
\hline
\end{tabular}

Table 2: Number of adverse reactions after proton therapy

\section{Discussion}

Ten articles were included in the study, and a total of 492 medulloblastoma patients underwent proton therapy. The results showed that the OS of proton therapy was higher than that of photon therapy. The study suggested that proton therapy was effective on OS and RFS for medulloblastoma patients.

Proton therapy can precisely plan the target area and cover the tumor foci, which protects normal brain tissue and ensures sufficient dose of radiation [5-7]. Meta-analysis showed that side effects of proton therapy included endocrine dysfunction, impaired speech processing speed and comprehension, hearing impairment.

Literature studies showed that proton group was superior to photon group in central/primary hypothyroidism $[8,9]$. Compared with photon therapy, the risk of sex hormone deficiency was apparently reduced (3\% vs. 19\%, $\mathrm{P}<0.01)$. The endocrine replacement therapy rate was obviously reduced ( $55 \%$ vs. $78 \%, \mathrm{P}<0.05$ ), while there was no significant difference in the incidence of growth hormone deficiency (53\% vs. 57\%), adrenal insufficiency (5\% vs. $8 \%$ ), or precocious puberty (18\% vs. 16\%)[6] $(\mathrm{P}>0.05)$.

Parents reported 59.1 health core scores, which was increased by 2.0 points per year [10]. These scores were significantly improved in proton therapy group, suggesting that proton therapy could continuously improve children's quality of life. The meta-analysis reported 1.5 point reduction per year in language processing speed and comprehension, while there was no significant difference in cognitive reasoning index and memory [11].

Benjamin J Moeller [12] had shown that hearing sensitivity decreased significantly in frequency ranges by proton therapy. The hearing threshold was partially reduced after radiotherapy, and the incidence of ototoxicity (grade 3 or 4 ) was $5 \%$ at 1 year after treatment.

This study has several limitations. First, the clinical effect of medulloblastoma in children was not evaluated by progression-free survival and recurrence survival, but by 5-year OS and RFS, and few literatures are available. Second, proton dose and fractionation were different in the study, including preoperative risk grade, resection degree, and chemotherapy, therefore, it was impossible to conduct a more detailed stratified comparative study. We will perform stratified analyses to pinpoint the independent prognostic factors and pathological characteristics which could affect the prognosis in medulloblastoma patients.Finally, different molecular types have different sensitivity and effectiveness to radiotherapy, and few studies have reported the metaanalysis to date. The influence of molecular types on radiotherapy efficacy should be paid more attention to subsequent case-control studies in the future.

To date, proton therapy is a safe and feasible method in medulloblastoma, which could improve the survival rate and RFS rate, and has fewer side effects than photon therapy. Clinical studies with high quality and a large sample size are needed before proton therapy is applied to medulloblastoma patients.

In brief, proton therapy is preferred radiotherapy for postoperative children with medulloblastoma.

\section{References}

1. Jiang T, Zhang Y, Wang J, Du J, Raynald, Qiu X, et al. A Retrospective Study of Progression-Free and Overall Survival in Pediatric Medulloblastoma Based on Molecular Subgroup Classification: A Single-Institution Experience. Front Neurol. 2017;8:198. Epub 2017/05/30. doi: 10.3389/fneur.2017.00198. PubMed PMID: 28553259; PubMed Central PMCID: PMCPMC5427081.

2. Chhabra A, Mahajan A. Treatment of common pediatric CNS malignancies with proton therapy. Chin Clin Oncol. 2016;5(4):49. Epub 2016/08/11. doi: 10.21037/ cco.2016.06.02. PubMed PMID: 27506805.

3. Goitein M, Cox JD. Should Randomized Clinical Trials Be Required for Proton Radiotherapy? Journal of Clinical Oncology. 2008;26(2):175-176. doi: 10.1200/ jco. 2007.14.4329.

4. Halperin EC. Randomized Prospective Trials of Innovative Radiotherapy Technology Are Necessary. Journal of the American College of Radiology. 2009; 6(1): 33-37. doi: 10.1016/j.jacr.2008.07.011.

5. Eaton BR, Esiashvili N, Kim S, Weyman EA, Thornton LT, Mazewski C, et al. Clinical Outcomes Among Children With Standard-Risk Medulloblastoma Treated With Proton and Photon Radiation Therapy: A Comparison of Disease Control and Overall Survival. Int J Radiat Oncol Biol Phys. 2016;94(1):133-138. Epub 2015/12/25. doi:10.1016/j.jirobp.2015.09.014. PubMed PMID: 26700707; PubMed Central PMCID: PMCPMC4692186.

6. Paulino AC, Mahajan A, Ye R, Grosshans DR, Fatih Okcu M, Su $\mathrm{J}$, et al. Ototoxicity and cochlear sparing in children with medulloblastoma: Proton vs. photon radiotherapy. Radiother Oncol. 2018;128(1):128-132. Epub 2018/01/27. doi: 10.1016/ j.radonc.2018.01.002. PubMed PMID: 29373195.

7. Jimenez RB, Sethi R, Depauw N, Pulsifer MB, Adams J, McBride SM, et al. Proton Radiation Therapy for Pediatric Medulloblastoma and Supratentorial Primitive Neuroectodermal Tumors: Outcomes for Very Young Children Treated With Upfront Chemotherapy. International Journal of Radiation Oncology, Biology, Physics. 2013; 87(1):120-126. doi: 10.1016/j.ijrobp.2013.05.017.

8. Eaton BR, Esiashvili N, Kim S, Patterson B, Weyman EA, Thornton LT, et al. Endocrine outcomes with proton and photon radiotherapy for standard risk medulloblastoma. Neuro Oncol. 2016;18(6):881-887. Epub 2015/12/22. doi: 10. 1093/ neuonc/nov302. PubMed PMID: 26688075; PubMed Central PMCID: PMCPMC 4864263.

9. Bielamowicz K, Okcu MF, Sonabend R, Paulino AC, Hilsenbeck SG, Dreyer Z, et al. Hypothyroidism after craniospinal irradiation with proton or photon therapy in patients with medulloblastoma. Pediatr Hematol Oncol. 2018;35(4):257-267. Epub 2018/12/13. doi: 10.1080/08880018.2018.1471111. PubMed PMID: 30537887.

10. Kamran SC, Goldberg SI, Kuhlthau KA, Lawell MP, Weyman EA, Gallotto SL, et al. Quality of life in patients with protontreated pediatric medulloblastoma: Results of a prospective assessment with 5-year follow-up. Cancer. 2018;124(16):33903400. Epub 2018/06/16. doi: 10.1002/cncr.31575. PubMed PMID: 29905942. 
11. Yock TI, Yeap BY, Ebb DH, Weyman E, Eaton BR, Sherry NA, et al. Long-term toxic effects of proton radiotherapy for paediatric medulloblastoma: a phase 2 single-arm study. The Lancet Oncology. 2016;17(3):287-298. doi: 10.1016/s1470-2045(15) 00167-9.
12. Moeller BJ, Chintagumpala M, Philip JJ, Grosshans DR, McAleer MF, Woo SY, et al. Low early ototoxicity rates for pediatric medulloblastoma patients treated with proton radiotherapy. Radiat Oncol. 2011;6:58. Epub 2011/06/04. doi: 10.1186/1748-717X-658. PubMed PMID: 21635776; PubMed Central PMCID: PMCPMC3123575.

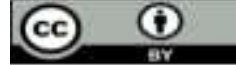

This work is licensed under Creative Commons Attribution 4.0 License

To Submit Your Article Click Here: Submit Manuscript

DOI: $10.31579 / 2578-8868 / 207$
Ready to submit your research? Choose Auctores and benefit from:

$>$ fast, convenient online submission

$>$ rigorous peer review by experienced research in your field

$>$ rapid publication on acceptance

$>$ authors retain copyrights

$>$ unique DOI for all articles

$>$ immediate, unrestricted online access

At Auctores, research is always in progress.

Learn more auctoresonline.org/journals/women-health-care-and-issues 